\title{
Genetic analysis and QTL mapping of maize yield and associate agronomic traits under semi-arid land condition
}

\author{
Jiufeng Guo ${ }^{1,3}$, Guoqin $\mathrm{Su}^{3}$, Jinpeng Zhang ${ }^{1}$ and Guoying Wang ${ }^{1,2 *}$ \\ ${ }^{1}$ State Key Laboratory of Agrobiotechnology, China Agricultural University, Beijing 100094, P.R. China. \\ ${ }^{2}$ Institute of Crop Sciences, Chinese Academy of Agricultural Sciences, Beijing 100081, P. R. China. \\ ${ }^{3}$ Biotechnology Centre, Inner Mongolia Academy of Agricultural Sciences, Huhhot 010031, P. R. China.
}

Accepted 16 May, 2008

\begin{abstract}
Drought is one of the major limiting factors in sustainable maize production all over the world. In order to develop maize variety with drought tolerance, it is necessary to explore the genetic basis and map the quantitative trait loci (QTLs) controlling the yield and associate agronomic traits under semi-arid land condition. In this study the QTLs for flower time, plant height, yield and yield components were characterized with recombinant inbred lines (RIL) derived from the cross $5003 \times$ p138 under drought stress and well-watered regime in the years 2004 and 2005, respectively. A linkage map was constructed based on 101 simple sequence repeat (SSR) markers covering a total of $1395.2 \mathrm{cM}$ genetic distance, and an average distance of $13.81 \mathrm{cM}$ between markers. A total of 51 QTLs were identified for 10 traits on 10 different chromosomes. Under the well-watered regime, 25 QTLs were identified for 9 traits, whereas under the water-stressed conditions, 22 QTLs were found for 7 traits. Four QTLs for drought tolerance index were found. Phenotypic variation associated with each QTL ranges from 1.68 to $13.3 \%$. The results reveal that most related traits cluster in chromosomal blocks: bnlg1614-bnlg1083 on chromosome 1 and bnlg1634-bnlg1209 on chromosome 9 for plant height, bnlg1035-bnlg1564 on chromosome 1 for yield and its components and drought insistence index, near NC012 and bnlg2907bnlg1136 on chromosome 6 for flower time and anthesis-silking interval (ASI), and near bnlg1063 on chromosome 5 and bnlg1241 on chromosome 4 for flower time. Four QTLs for male flower time were identified in marker bnlg1327-bnlg1812 on chromosome 8 under two water regimes. These QTLs may be useful for the marker-assisted selection in maize breeding for drought resistance.
\end{abstract}

Key words: Maize, drought resistance, genetic mapping, SSR.

\section{INTRODUCTION}

Maize is one of the main crops, and as such is a very important crop for food security in China. About $2 / 3$ of maize produced in China is cultivated in the arid or semiarid areas. Drought or water stress is the main environmental factor causing substantial yield reductions, which was estimated between 9.3 and $15.5 \%$ every year (Wang et al., 2006). In order to increase maize production, maize breeders have exerted enormous efforts to breed hybrids with drought tolerance (Bruce et al., 2002).

However, because the genetic mechanism of drought tolerance in maize is very complex, the yield trait is

\footnotetext{
*Corresponding author. E-mail: gywang@caas.net.cn.
}

strongly influenced by both genotype and environment, and the interaction of genotype-by-environment is remarkable. Traditional breeding programs that depend on phenotype selection are time-consuming and less efficient (Andjelkovic and Thompson, 2006; Duvick, et al., 2004; Loo, 1991). At present, more powerful and efficient strategies for producing 'ideal' crops are possible with the development of molecular biology (Ribaut et al., 1997). For instance, the QTL pyramiding approach results from a combination of recent crop genomics and conventional breeding, which promotes better crop breeding. Applying molecular marker techniques on traditional breeding programs can improve the efficiency of the breeding of drought-tolerant maize (Tuberosa et al., 2002). It is generally recognized that complex traits can be dissected 
as Mendelian factors with molecular markers (Paterson, 1988). The identification of quantitative trait loci (QTL) is the basis of molecular marker-assisted selection (Eathington, 1997; Hoisington et al., 1996; Yu et al., 2005). Discovery of drought tolerant genes and their closely linked markers can provide useful information in setting up molecular breeding strategies. So the biotechnological work has focused on the genetic dissection of drought tolerance through the identification of QTL associated with yield components as well as secondary morphological traits of interest (e.g., anthesis-silking interval, plant height). The introduction of DNA-based molecular markers not only allows for the identification of QTL that determines the phenotypic value of particular trait, it is also useful in the analysis and interpretation of the cause-effect relationship among traits (Lebreton et al., 1995). During the past decade, major efforts have been dedicated to the genetic dissection of drought tolerance components in maize under water stress conditions (Guo et al., 2004; Jeanneau et al., 2002; Quan et al., 2004; Veldboom, 1996). A lot of QTLs involved in the yield components and secondary morphological traits of interest, such as anthesis-silking interval (ASI), had been identified (Agrama, 1996; Campos, 2004; Xiao et al., 2004). QTLs that control grain yield under drought stress had been studied and 5 QTLs were detected. Ribaut et al. (1997) examined the QTLs that govern the yield of tropical maize under 3 irrigation regimes: a total of 5 and 4 QTLs were detected under intermediate and severe stresses, respectively (Ribaut et al., 1997). Austin (1996) evaluated the same population at the F6:7 generation under more stressful conditions and detected $35 \%$ of the QTLs for morphological traits in both the stress and non-stress environments. These studies suggest that environmental factors can greatly affect the perception of QTLs (Austin, 1996; Frova et al., 1999; Sari-Gorla et al., 1999; Zhang et al., 2006). Li et al. (2005) used maize genomic database to explore the QTLs relevant to drought tolerance in maize. A total of 181 QTL involved in the expression of 9 morphophysiological traits in 10 mapping populations of maize under drought conditions were screened out, and only 15 "universal drought tolerance QTL" and their linked markers were identified.

China is one of the main maize production countries, but only a few QTLs were identified under water stressed conditions (Li et al., 2004). Because of the complexity of the physiological pathways of both yield and drought tolerance, it is necessary to identify the QTLs based on more mapping populations and accumulate more QTLs data in order to understand the mechanism of QTL well and lay a foundation for its application. The aims of this study are to identify the QTLs relevant to yield and yield components, analyze the genetic effects, determine the number, genomic positions, and gene effects of QTLs involved in the variation of grain yield (GY), plant height $(\mathrm{PH})$, ear height $(\mathrm{EH})$, and reveal the relationships among the QTLs under water stress condition, which will be helpful for the maize improvement of drought resistance in the future.

\section{MATERIALS AND METHODS}

\section{Plant materials}

A total of 450 maize RILs (recombinant inbred lines, F6:7) randomly selected from a population derived from the hybrid $5003 \times$ p 138 were used in this study. The two parental lines were also included in the field evaluation. The drought tolerance of the female parent 5003 was better than that of the male parent p138 according to experiments performed over the past few years by our laboratory. A total of 116 pairs of SSR markers were selected based on our previous work (unpublished).

\section{Field conditions}

The trial was carried out in the experimental farm located at the Inner Mongolia Academy of Agricultural Sciences in 2004 and 2005 , which is located in the Northwest of China. Rainfall during maize growing season is usually very limited so water supply is easily controlled by irrigation. It is a region that is typically suitable for the evaluation of the drought tolerance of maize. Two levels of water treatment were imposed, which were the well watered (WW) and water stressed (WS) conditions. A random complete design with 3 replicates under WW and WS for each block was made. Plots consisted of single rows, $0.5 \mathrm{~m}$ apart and $6 \mathrm{~m}$ long. A total of 15 plants per genotype were grown at a row, with a plant density equal to $0.5 \times 0.4$ square meter. All blocks were well-irrigated before planting. The seeds were sown on April 20, 2004 and 2005. During the growing season, the well watered plots received 3 irrigations, while the stressed blocks received just one time of irrigation in order to keep the plants alive. Male flowering time (MFT), female flowering time (FFT), anthesis-silking interval (ASI) and plant height $(\mathrm{PH})$ were recorded in terms of the number of days for the tassel glumes (MFT) or visible silks (FFT) in $50 \%$ of the plants per plot. Plant height was measured (in $\mathrm{cm}$ ) from the ground surface to the tip of the tassel after the flowering was completed. Measurement was performed on five plants per plot. A total of 10 centrally located plants per plot were harvested on September 20, 2004 and 2005. Ear length $(E L)$, ear weight $(E W)$, ear diameter $(E D)$, kernel row number per ear (KRN), kernel yield per plot (GY), and 100-kernel weight $(100 \mathrm{KW})$ were determined after drying.

\section{Analysis of phenotypic data}

Plot means, range of means, and standard errors were calculated for individual years on the complete unadjusted data set, keeping the replications separate. A drought tolerance index (TI) was calculated as $T / C \times 100$, where $T$ is the GY under stress, and $C$ represents the GY evaluated under well watered condition. Coefficient of variation (CV) is calculated as $\mathrm{sd} / \mathrm{mean} \times 100$. The statistical analysis (ANOVA etc) of phenotypic traits was carried out using Microsoft Office Excel 2003. Simple correlation coefficients among traits in the family within the same water regime were estimated by using the PROC CORRPEARSON option.

\section{Mapmaker and QTL detection}

DNA extraction, PCR reaction, gel electrophoresis, and silver staining were performed following the protocol described by George 
(2004). The SSR markers showing polymorphisms between parents were used to genotype the 450 maize RILs and their segregation ratios were tested by $x^{2}$ goodness-of-fittest. Only the SSR markers showing a segregation ratio of the expected Mendelian segregation ratio were used to create a genetic map. Linkage construction of SSR markers was conducted by multipoint analysis using the computer program MAPMAKER (version 3.0; $[\mathrm{LOD}]=3.0, r=0.4$ ). QTL identification was done by composite interval mapping method (CIM) according to Zeng (1994) using the WinQTL cartographer version 2.5 version software (Wang et al., 2005). Model 6 of the QTL cartographer module was used; intervals of $1 \mathrm{cM}$ between markers and putative QTLs with a window size of $10 \mathrm{cM}$ were scanned. The number of marker cofactors for background control was set by forward-backward step wise regression.

\section{RESULTS}

\section{Phenotypic data analysis}

All the traits revealed a large quantitative variability and a fairly normal frequency distribution in both water regimes conditions, as shown in Figure 1. The two parental lines were clearly differentiated for all traits especially in $\mathrm{PH}$ and GY. The inbred p138 was taller than 5003. Water stress reduced the plant height more in p138 than in 5003 under drought conditions; the RILs population showed a general shift of the distributions towards lower trait values. Thus, this population was suitable for QTL analysis of drought tolerance. The traits of the RIL population and its parents under two different water trials are given in Table 1. The average value of yield and its components decreased under water stressed conditions compared with well watered conditions. The '5003' and 'P138' lines differed in MFT and FFT, under water stress; 5003 had a smaller ASI than p138.

\section{Statistical analysis of all traits}

All traits in this experiment had highly significant differences among the lines and highly significant genotype $\times$ environment interaction as shown in Table 2. Correlations among GY, EL, ED, $100 \mathrm{KW}$ and $\mathrm{PH}$ under the WS regime were positive and statistically significant (Table 3 ). Correlation between $\mathrm{TI}$ and $\mathrm{ASI}$ was negative and statistically significant. Phenotypic correlations among all traits under the WW regime were similar to those under the WS regime. Linear regression of the population drought-tolerance index (TI) with ASI in water stress conditions gave a highly significant negative coefficient of regression. A high value of the index for $G Y$ indicates a greater tolerance (small reduction in GY under stress).

\section{Construction of linkage map and QTL analysis}

A total of 101 SSR markers between 2 parental lines were polymorphic and fitted to the expected Mendelian segregation ratio. These markers were assigned to 10 linkage groups, covered $1395.2 \mathrm{cM}$, and with an average interval of $13.81 \mathrm{cM}$. A total of 51 significant QTLs were identified throughout the two year experiment, and all traits are summarized in Table 3 . Two to ten QTLs were detected for each trait. Each QTL explained phenotypic variations ranging from 1.68 to $13.3 \%$. Most of the QTLs were located in the same or adjacent regions as those reported in previous studies. They were distributed on all of the chromosomes, and showed clustering in several chromosomal regions (Figure 2).

\section{QTLs for plant height}

A total of three QTLs for plant height were detected on chromosome 4 and 10 (Table 3) under well watered condition. They can explain $8.0 \%$ of the variation in these loci: two alleles had additive effects associated with '5003', while one allele increased the plant height value for ' $p 138$ '. Seven QTLs were detected for plant height on chromosome 1, 3, and 9 under water stress. This accounted for $17.4 \%$ of the variation. There were 5 loci exerting an additive effect associated with ' $p 138$ ', with two of them coming from ' 5003 '. This means that the plant height of the population depended on '5003' in well water and a great part of variation was caused by ' $\mathrm{p} 138$ ' under water stressed regime.

\section{QTLs for male flowering time}

Eight QTLs conferring expression of male flowering time were detected on chromosomes 2, 4, 5, 6, and 8 under two regime conditions (Table 5). Four QTLs were located on chromosomes 2, 6, and 8 under well watered condition, accounting for $18.72 \%$ of the phenotypic variation. Two of them displayed positive additive effect while the rest displayed negative additive effects. The additive effect originated from the two parents. Four QTLs were located on chromosomes 4,5 , and 8 under water stress condition. They can account for $23.05 \%$ of the phenotypic variation. All displayed positive additive effects imply that the additive effect originated from parent ' $p 138$ '. Four QTLs were identified on chromosomes 8 between bnlg1327 and bnlg1812 under WW and WS regimes, respectively.

\section{QTLs for female flowering time}

Six QTLs with significant additive effects for female flowering time were found on chromosomes 2, 4, 6, and 10 under well-watered condition, which could account for $17.82 \%$ of the phenotypic variation. Among these loci, the '5003' allele showed an increasing additive effect of four loci. Two of these loci originated from ' $p 138$ '. Three QTLs on chromosomes 5 and 9 were detected under the stressed regime, which could account for $7.96 \%$ of the 

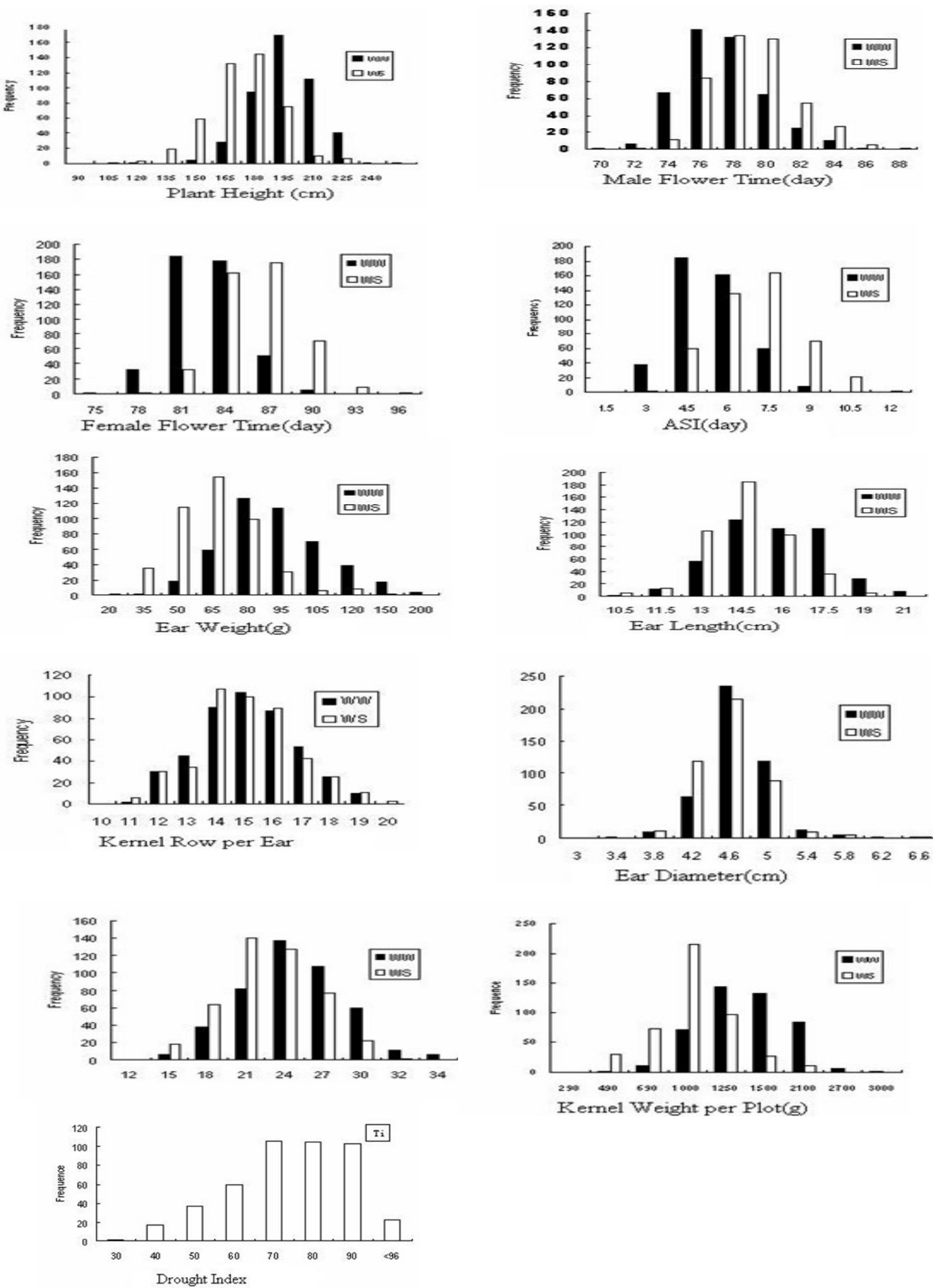

Figure 1. Frequency distribution of trait in maize RIL population. 
Table 1. Variation of traits in maize RIL population.

\begin{tabular}{|l|c|c|c|c|c|c|c|c|}
\hline \multirow{2}{*}{ Trait } & \multicolumn{2}{|c|}{ RIL population (mean \pm SD) } & \multicolumn{2}{c|}{ p1 (mean) } & \multicolumn{2}{c|}{ p2 (mean) } & \multicolumn{2}{c|}{ CV (\%) } \\
\cline { 2 - 9 } & WW & WS & WW & WS & WW & WS & WW & WS \\
\hline PH (cm) & $175.59 \pm 16.42$ & $152.71 \pm 18.00$ & 160.22 & 140.13 & 190.47 & 160.73 & 9.35 & 11.79 \\
MFT (day) & $76.63 \pm 2.43$ & $78.32 \pm 2.51$ & 80.61 & 82.32 & 74.37 & 75.44 & 3.71 & 3.20 \\
FFT (day) & $81.51 \pm 2.33$ & $84.84 \pm 2.60$ & 83.52 & 87.33 & 81.29 & 86.32 & 2.86 & 3.06 \\
ASI (day) & $4.88 \pm 1.23$ & $6.52 \pm 1.55$ & 4.12 & 5.16 & 7.11 & 10.31 & 25.20 & 23.72 \\
EL (cm) & $15.01 \pm 1.91$ & $13.93 \pm 1.47$ & 12.22 & 12.45 & 18.36 & 13.31 & 12.73 & 10.57 \\
ED (cm) & $4.52 \pm 0.36$ & $4.42 \pm 0.35$ & 4.34 & 3.82 & 4.62 & 4.03 & 7.87 & 8.02 \\
EW (g) & $112.93 \pm 27.40$ & $82.95 \pm 21.49$ & 80.15 & 65.37 & 150.27 & 85.43 & 24.26 & 25.90 \\
KRN & $15.05 \pm 1.69$ & $15.01 \pm 1.74$ & 16.02 & 16.23 & 16.01 & 14.17 & 11.25 & 11.61 \\
100KW (g) & $23.33 \pm 3.88$ & $21.38 \pm 3.50$ & 18.15 & 17.42 & 27.11 & 25.27 & 16.61 & 16.38 \\
GY (g) & $1270.16 \pm 368.99$ & $875.89 \pm 322.88$ & 900.41 & 720.62 & 1750.54 & 900.19 & 29.05 & 36.86 \\
\hline
\end{tabular}

Table 2. ANOVA of drought-related traits in maize RIL population.

\begin{tabular}{|l|l|l|l|l|l|l|l|l|l|l|}
\hline Parameter & \multicolumn{1}{|c|}{ EW } & EL & ED & KRN & GY & 100GW & MF & FF & ASI & PH \\
\hline Lines & $8.31^{* *}$ & $2.172^{* *}$ & $1.526^{* *}$ & $3.035^{* *}$ & $10.709^{* *}$ & $11.839^{* *}$ & $6.723^{* *}$ & $6.530^{* *}$ & $5.519^{* *}$ & $20.063^{* *}$ \\
Regime & $1701.34^{* *}$ & $172.289^{* *}$ & $22.30^{* *}$ & 0.623 & $2622.0^{* *}$ & $601.8^{* *}$ & $446.09^{* *}$ & $1716.0^{* *}$ & $1155.4^{* *}$ & $4880.3^{* *}$ \\
Interaction & $1.86^{* *}$ & $1.156^{* *}$ & 1.029 & $1.281^{*}$ & $2.001^{* *}$ & $7.469^{* *}$ & $1.685^{* *}$ & $1.846^{* *}$ & $1.954^{* *}$ & $4.531^{* *}$ \\
\hline
\end{tabular}

** ** Significant at $\mathrm{P}=0.05$ and $\mathrm{P}=0.01$, respectively.

Table 3. Correlation among traits in maize RIL population under WW and WS regime.

\begin{tabular}{|l|c|c|c|c|c|c|c|c|c|c|}
\hline & EW & EL & ED & KRN & MFT & FFT & ASI & PH & 100KW & GY \\
\hline EW & 1 & 0.601 & 0.347 & 0.048 & -0.051 & -0.054 & -0.003 & 0.128 & 0.275 & 0.954 \\
EL & 0.322 & 1 & 0.350 & -0.063 & -0.094 & -0.062 & 0.068 & 0.058 & 0.332 & 0.586 \\
ED & 0.237 & 0.378 & 1 & 0.199 & -0.018 & 0.033 & 0.098 & -0.023 & 0.174 & 0.319 \\
KRN & 0.089 & 0.024 & 0.201 & 1 & 0.052 & 0.003 & -0.097 & -0.069 & -0.191 & 0.060 \\
MFT & -0.015 & 0.027 & 0.051 & 0.054 & 1 & 0.867 & -0.327 & 0.083 & -0.201 & -0.065 \\
FFT & -0.073 & 0.016 & 0.049 & 0.041 & 0.817 & 1 & 0.187 & 0.074 & -0.145 & -0.067 \\
ASI & -0.099 & -0.017 & -0.001 & -0.019 & -0.248 & 0.355 & 1 & -0.024 & 0.121 & 0.002 \\
PH & 0.169 & 0.101 & 0.008 & 0.014 & 0.165 & 0.165 & 0.010 & 1 & 0.061 & 0.131 \\
100KW & 0.322 & 0.125 & -0.013 & -0.127 & -0.100 & -0.085 & 0.019 & 0.010 & 1 & 0.280 \\
GY & 0.964 & 0.329 & 0.237 & 0.098 & -0.014 & -0.070 & -0.095 & 0.166 & 0.354 & 1 \\
TI & 0.520 & 0.227 & 0.138 & 0.063 & 0.116 & 0.014 & -0.165 & 0.084 & 0.158 & 0.534 \\
\hline
\end{tabular}

The data at upper diagonal portion were water well treated, down diagonal portion, water stress treated.

$r_{0.05}=0.088, r_{0.01}=0.116$.

phenotypic variation. The loci FMM 3 on chromosome 6 near the marker NC012 had been detected in both well watered and water stress conditions. In general, '5003' exerted a greater effect in female flowering time than 'p138'.

\section{QTLs for anthesis-silking interval (ASI)}

Four QTLs conferring ASI were identified on chromosomes 3,6 , and 7 under well-watered regime, which showed additive effects of $0.10,-0.12,0.28$ and -0.40 , respectively. Two QTLs were found to be associated with '5003', which could account for $11.58 \%$ of the phenotypic variation. Others were found to be associated with ' $p 138$ ', with an additive effect of 0.38 , accounting for $4.71 \%$ of the phenotypic variation. Two weak QTLs were mapped on chromosomes 5 and 6 with additive effects of 0.16 and -0.36 under water stress condition, accounting for 2.85 and $5.34 \%$ of the phenotypic variation, respectively. The loci ASI1 and ASI6, all near the marker bnlg1136 on chromosomes 6 , can be detected in the two regimes, 

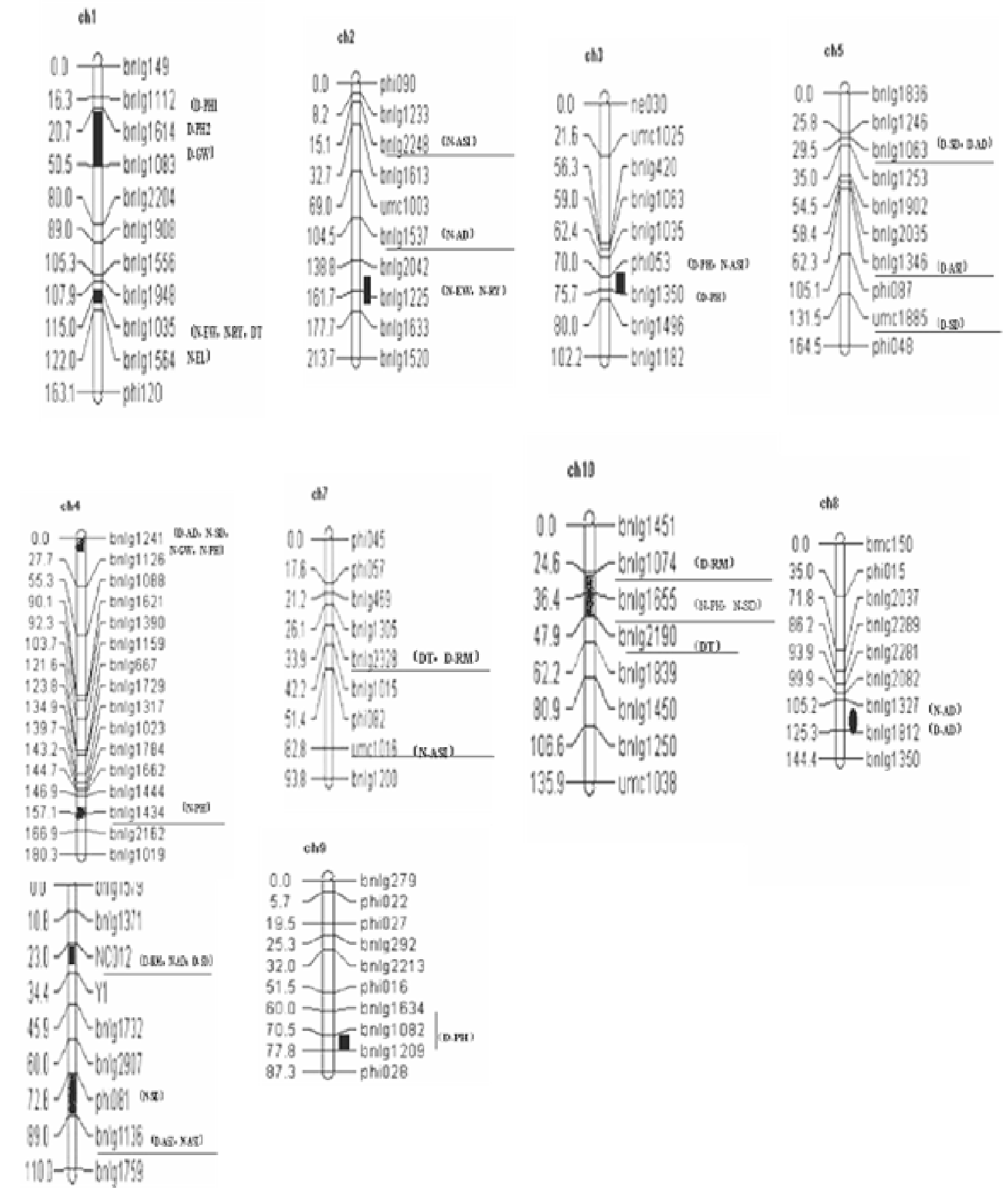

all
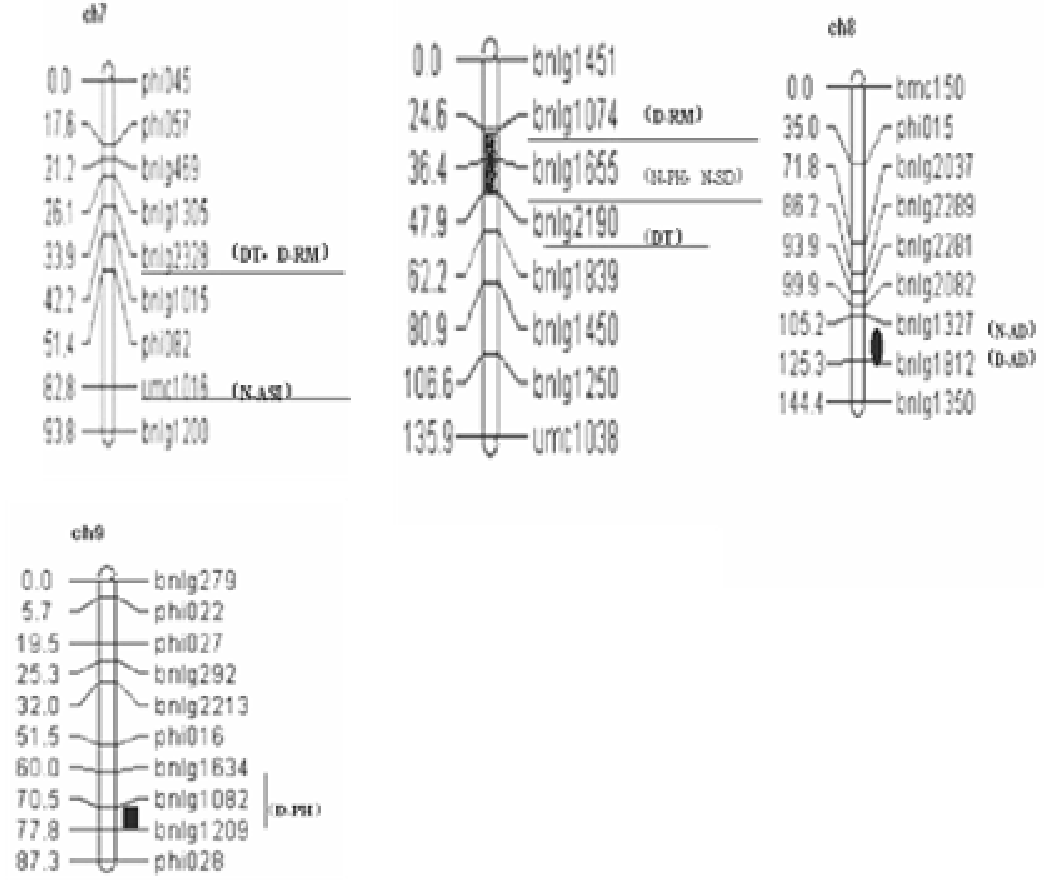

Figure 2. The position of QTLs for drought tolerance traits in RIL linkage groups. D: drought treat; N: water well treated; ASI: anthesis-silking interval; AD: Male flowering time; DT: drought tolerance index; EL: Ear length; EW: ear weight; GW: 100 -kernel weight; PH: plant height; RM: kernel row number per ear; RY: Kernel yield per plot; SD: female flowering time.

which means that the loci had stable heredity.

\section{QTLs for ear weight}

In the well watered regime, two QTLs were detected for ear weight on chromosomes 1 and 2 with additive effects of -4.52 and -4.04 , explaining 2.73 and $2.19 \%$ of the phenotypic variance, respectively. No significant QTL for ear weight was identified under the WS regime.

\section{QTLs for ear length}

Like ear weight, two QTLs for ear length were detected on chromosome 1 with additive effect of -0.35 and -0.31 
Table 4. QTL for drought -related traits in maize RIL population.

\begin{tabular}{|c|c|c|c|c|c|}
\hline Trait & Chrom & Adjacent Marker & LOD & Additive & $\mathbf{R}^{2}$ \\
\hline \multicolumn{6}{|c|}{ Plant height } \\
\hline W-ph1 & 4 & bnlg1434 & 2.45 & -2.75 & 2.38 \\
\hline W-ph2 & 4 & bnlg1241 & 1.96 & -3.23 & 3.66 \\
\hline W-ph3 & 10 & bnlg1655 & 1.73 & 2.30 & 1.94 \\
\hline S-ph1 & 1 & bnlg1614 & 2.27 & 2.05 & 2.69 \\
\hline S-ph2 & 1 & bnlg1083 & 2.48 & 2.53 & 4.12 \\
\hline S-ph3 & 3 & phi053 & 1.70 & -1.62 & 1.68 \\
\hline S-ph4 & 3 & phi053 & 1.87 & -2.55 & 1.97 \\
\hline S-ph5 & 9 & bnlg1209 & 1.87 & 2.64 & 2.13 \\
\hline S-ph6 & 9 & bnlg1634 & 2.29 & 2.74 & 2.31 \\
\hline S-ph7 & 9 & bnlg1082 & 2.37 & 2.90 & 2.57 \\
\hline \multicolumn{6}{|c|}{ Male flower time } \\
\hline W-mf1 & 2 & bnlg1537 & 2.21 & -0.52 & 4.48 \\
\hline W-mf2 & 6 & $\mathrm{nc012}$ & 2.04 & -0.45 & 2.57 \\
\hline W-mf3 & 8 & bnlg1327 & 2.01 & 0.43 & 2.12 \\
\hline W-mf4 & 8 & bnlg1812 & 2.08 & 0.84 & 9.55 \\
\hline S-mf1 & 4 & bnlg1241 & 1.89 & 0.51 & 3.46 \\
\hline S-mf2 & 5 & bnlg1063 & 2.29 & 0.78 & 3.28 \\
\hline S-mf3 & 8 & bnlg1812 & 2.58 & 1.03 & 13.30 \\
\hline S-mf4 & 8 & bnlg1327 & 2.40 & 0.52 & 3.01 \\
\hline \multicolumn{6}{|c|}{ Female flower time } \\
\hline W-ff1 & 2 & bnlg1537 & 2.18 & -0.37 & 2.52 \\
\hline W-ff2 & 6 & bnlg2907 & 2.34 & -0.43 & 3.32 \\
\hline W-ff3 & 6 & phi081 & 2.19 & -0.47 & 4.02 \\
\hline W-ff4 & 6 & nc 012 & 1.83 & -0.43 & 2.32 \\
\hline W-ff5 & 10 & bnlg1655 & 1.91 & 0.36 & 2.39 \\
\hline W-ff6 & 4 & bnlg1241 & 1.77 & 0.48 & 3.25 \\
\hline S-ff1 & 5 & bnlg185 & 1.65 & -0.50 & 3.59 \\
\hline S-ff2 & 5 & bnlg1063 & 2.02 & 0.37 & 1.97 \\
\hline S-ff3 & 6 & $\mathrm{nc012}$ & 1.87 & -0.50 & 2.40 \\
\hline \multicolumn{6}{|c|}{ Anthesis-silking interval } \\
\hline W-asi1 & 2 & bnlg2248 & 1.74 & 0.10 & 1.69 \\
\hline W-asi2 & 3 & phi053 & 3.10 & 0.28 & 3.02 \\
\hline W-asi3 & 6 & bnlg1136 & 2.61 & -0.40 & 9.07 \\
\hline W-asi4 & 7 & umc1016 & 2.38 & -0.12 & 2.51 \\
\hline S-asi1 & 5 & bnlg1346 & 1.86 & 0.16 & 2.85 \\
\hline S-asi2 & 6 & bnlg1136 & 1.68 & -0.36 & 5.34 \\
\hline \multicolumn{6}{|c|}{ Kernel yield per plot } \\
\hline W-gy1 & 1 & bnlg1035 & 2.50 & -3.84 & 2.93 \\
\hline W-gy2 & 1 & bnlg1564 & 2.06 & -3.47 & 2.41 \\
\hline W-gy3 & 2 & bnlg1225 & 2.06 & -3.24 & 2.19 \\
\hline S- gy1 & 1 & bnlg1948 & 1.60 & 19.23 & 2.59 \\
\hline S- gy2 & 9 & phi022 & 1.62 & -17.31 & 2.11 \\
\hline \multicolumn{6}{|c|}{ Ear length } \\
\hline W-el1 & 1 & bnlg1035 & 2.25 & -0.35 & 2.78 \\
\hline W-el2 & 1 & bnlg1564 & 1.86 & -0.32 & 2.29 \\
\hline \multicolumn{6}{|c|}{ Kernel rows per ear } \\
\hline S-krn1 & 6 & $\mathrm{nc012}$ & 1.76 & -0.23 & 1.68 \\
\hline S-krn2 & 7 & bnlg2328 & 1.87 & -0.25 & 2.04 \\
\hline S-krn3 & 10 & bnlg2042 & 1.72 & -0.25 & 2.08 \\
\hline
\end{tabular}


Table 4. Contd.

\begin{tabular}{|l|c|l|l|l|l|}
\hline Ear weight & $\begin{array}{l}\text { W-ew1 } \\
\text { W-ew2 }\end{array}$ & bnlg1035 & $\begin{array}{l}2.44 \\
\text { bnlg1225 }\end{array}$ & $\begin{array}{r}-4.53 \\
-4.04\end{array}$ & 2.73 \\
\hline \multicolumn{7}{|l|}{} \\
\hline 100 kernel weight
\end{tabular}

in well watered regime, explaining 2.78 and $2.29 \%$ of the phenotypic variance, respectively. No significant QTL for ear length was identified under the WS regime.

\section{QTLs for kernel row number}

Three QTLs for kernel rows were detected under water stress condition. They were located on chromosomes 6 , 7 , and 10 with negative additive effect of $-0.23,-0.25$ and 0.25 respectively, accounting for $5.7 \%$ of phenotypic variance. No significant QTLs for grain rows were found in well watered condition. It showed that for grain row trait the additive effect originated from parent '5003'.

\section{QTLs for $100 \mathrm{KW}$}

Two QTLs were identified under WW and WS regimes. The QTL on chromosome 1 near bnlg1083 had a positive additive effect of 0.4968 under water stress condition, accounting for $2.0 \%$ of phenotypic variance. One QTL on chromosome 4 near bnlg1241 exerted a negative additive effect of -1.8967 under well watered condition, accounting for $3.8 \%$ of phenotypic variance. These show that the parent ' $\mathrm{p} 138$ ' is associated with $100 \mathrm{KW}$ under water stress regime.

\section{QTL for drought tolerance index (Ti)}

A total of four QTLs were mapped on chromosomes 1, 7 and 10. Two loci were on chromosomes 7 and 10 near bnlg 2328 and bnlg 2190, respectively; they had a negative additive effect, explaining $3.6 \%$ of the phenotypic variance in 2004. Two QTLs were on chromosome 1 near bnlg1035 and bnlg1564 respectively with a positive additive effect accounting for $7.7 \%$ of the phenotypic variance in 2005. This means that the drought tolerance of yield was complex. The time and intensity of water stress have difference effects on the yield of maize.

\section{QTLs for kernel yield per plot}

Three QTLs for plot yield were detected on chromosomes 1 and 2 under well watered condition. The additive effects were $-3.84,-3.47$, and -3.24 respectively, explaining $7.5 \%$ of the phenotypic variance. All loci with additive effect were derived from '5003'. Two weak QTLs for the plot yield were detected on chromosomes 1 and 9 under water stress condition, accounting for $4.7 \%$ of the variation. One locus was from 'p138' and another from '5003'.

\section{DISCUSSION}

Obtaining a high grain yield is the most important goal in maize breeding programs, but its evaluation and improvement are difficult and expensive because of the maize's complex biology, environmental interactions, and low heritability. Indirect selection through secondary traits associated with grain yield can improve the efficiency of the breeding for drought-tolerant strains. Understanding the genetic basis of drought tolerance in maize is fundamental to enable the breeder to develop new methods of selection. The introduction of DNA-based molecular markers not only allows for the identification of QTL that determines the phenotypic value of particular trait, but also for the analysis and interpretation of the cause-effect relationship among traits. It is generally recognized that complex traits can be dissected as Mendelian factors with molecular markers according to Paterson et al. (1988). So it is necessary to identify the QTLs for yield components and morphological traits in more populations, different locations and under different stress regimes.

\section{Analysis of effect per QTL}

Only a few QTLs with sharp effects were detected in this study, possibly because only stable QTLs across environments are being mapping in this research. For all the characters, the majority of the QTLs detected had 
small effects, explaining less than $10 \%$ of the phenotypic variation. Only a QTL for MFT exerted a considerably higher effect, explaining $13.3 \%$ of the variation. This result is mainly due to a QTL which exerted dominant effects several times greater than the corresponding additive effects. This kind of gene action may reflect the effects of several QTLs within the genomic interval under study, a situation that would tend to result in overestimation of dominance (Sibov et al., 2003). RIL has its advantage; RIL families are more suitable for mapping population because they are genetically fixed, so we can repeat the experiment with the same population, and at the same time minor QTLs can be detected. But the RIL population had a disadvantage, i.e., QTL analyses were based on the additive genetic model. The dominant effect and interactive effect among alleles cannot be determined because the additive and epistatic QTLs exist at the same time, and the major and minor QTLs all contribute to the phenotype under water stressed conditions.

The use of marker-assisted selection opens up viable prospects for new strategies in breeding. To a large extent, traditional breeding efforts for drought tolerance depended on field tests of progenies, and accurate field evaluation is often laborious and difficult (Ribaut et al., 1997). Marker-assisted selection, which enables breeders to distinguish genotypes directly, can be a better approach to develop drought-tolerant germ plasm, a complex trait influenced by multiple genes.

There are about $2.5 \times 10^{6}$ base pairs are contained in maize genome, averaging $1000 \mathrm{~kb}$ per $\mathrm{cM}$, so there is a gap between gene clone and QTL in preliminary mapping population. A more common goal of quantitative trait mapping is the use of QTL in marker-assisted selection (MAS) to transfer genomic regions associated with drought tolerance from a tolerant source to an elite but drought-susceptible genotype, thus augmenting and enhancing the outcomes of phenotypic selection. Johnson (2004) and Ribaut et al. (1997) described the transfer of five QTL from donor line Ac7643 into the drought susceptible recipient line CML 247. The transferred fragments included $12 \%$ of the genome containing these QTL and a further 7\% lying outside of these regions (Johnson, 2004). Correlation between TI and ASI was negative and statistically significant in this study. These QTLs have potential use in molecular markerassisted selection. We can expect that shortening the ASI using marker-assisted selection should result in improvements that will render tolerance to drought.

In maize, there have been several reports on QTLS associated with specific phenotypes observed under drought stress in diverse mapping populations (Veldboom and Lee, 1996; LeDeaux et al., 2006; Ribaut et al., 1996, 1997b; Frova et al., 1999; Tuberosa et al., 2002). Frova et al. (1999) identified 17 QTLs for yield components; more than $50 \%$ of them were also detected under well watered (WW) conditions, and some of them were common to 2 or more traits. About 74 QTLs for grain yield and more for yield components have been detected (Maize Genetics and Genomics Database, 2003). For grain yield, the QTLs were observed on chromosomes 3 and 8 only. For ASI, QTLs were observed on chromosomes 1, 2, 6, 8, and 10. In contrast to chromosome 1 , the QTLs on chromosome 10 had a significant effect on both GY and ASI.

A clustering of QTL for drought related traits in specific chromosomal regions is apparent. In this study, most QTLs are only detected under water stress or well watered condition. However, some QTL for MFT and ASI have been identified at the same loci under both conditions. These results seem to confirm the fact that the QTLs are normally located in clusters which contain genes that control development (Khavkin and Coe, 1998). A total of 51 QTLs were identified for ten traits on ten different chromosomes in this study. The results revealed that QTLs for related traits clustered in chromosomal blocks, for example, bnlg1614-bnlg1083 on chromosome 1 and bnlg1634-bnlg1209 on chromosome 9 for plant height. QTLs for yield and its components and drought tolerance index were detected between bnlg1035-bnlg1564 on chromosome 1 near NC012; flower time QTLs were found at bnlg2907-bnlg1136 on chromosome 6; ASI, near bnlg1063 on chromosome 5; and bnlg1241 on chromosome 4 for flower time. Four QTLs for male flower time were identified between markers bnlg1327-bnlg1812 on chromosome 8 and two QTLs for ASI on chromosome 6 under two water regimes. These QTLs may be useful for maize molecular marker-assisted breeding for drought tolerance.

\section{ACKNOWLEDGEMENT}

This work is supported by the National Natural Science Foundation of China (30730063).

\section{REFERENCES}

Agrama HMM (1996). Mapping QTLs in breeding for drought tolerance in maize (Zea mays L.). Euphytica, 91: 89-97.

Andjelkovic V, Thompson R (2006). Changes in gene expression in maize kernel in response to water and salt stress. Plant Cell Rep, 25: 71-79.

Austin DFLM (1996). Comparative mapping in F-2:3 and F-6:7 generations ofquantitative trait loci for grain yield and yield components in maize. Theor. Appl. Genet. 92: 817-826.

Bruce WB, Edmeades GO, Barker TC (2002). Molecular and physiological approaches to maize improvement for drought tolerance. $\mathrm{J}$. Exp. Bot. 53: 13-25.

Campos HM (2004). Improving drought tolerance in maize:a view from industry. Field Crops Res. 90: 19-34.

Duvick DN, Smith JSC, Cooper M (2004). Long-term selection in a commercial hybrid maize breeding program. Plant Breed Rev. 24: 109-151.

Eathington SR (1997). Usefulness of Marker-QTL Association in Early Generation Selection. Crop Sci., 37: 1686-1693.

Frova C, Krajewski P, Fonzo ND, Villa M, Sari-Gorla M (1999). Genetic analysis of drought tolerance in maize by molecular markers. I. Yield components. Theor. Appl. Genet. 99: 280-288. 
George M (2004). Laboratory Handbook Protocols for Maize Genotyping using SSR Markers and Data Analysis,AMBIONET Service Laboratory, CIMMYT.

Guo M, Rupe MA, Zinselmeier C, Habben J, Bowen BA, Smith OS (2004). Allelic variation of gene expression in maize hybrids. Plant Cell, 16: 1707-1716.

Hoisington D, Jiang C, Khairallah M, Ribaut JM, Bohn M, Melchinger A (1996). QTL for insect resistance and drought tolerance in tropical maize: prospects for marker assisted selection. Symp. Soc. Exp. Biol. 50: 39-44.

Jeanneau M, Gerentes D, Foueillassar X, Zivy M, Vidal J, Toppan A (2002). Improvement of drought tolerance in maize: towards the functional validation of the Zm-Asr1 gene and increase of water use efficiency by over-expressing C4-PEPC. Biochimie, 84: 1127-1135.

Johnson R (2004). Marker-assisted selection. Plant Breed Rev., 24: 239-309.

Li Y, Wang T, Shi Y, Song Y (2004). Advances and prospects on QTL analysis of drought tolerance of maize (Zea mays L.). Agric. Res. Arid Areas 22: 32-39.

Li X-H, LI X-H, Hao Z-F, Tian Q-Z, Zang S-H (2005). Consensus Map of the QTL Relevant to Drought Tolerance of Maize Under Drought Conditions. Sci. Agric. Sinic. 38: 882-890.

Lebreton C, LazicJancic V, Steed A, Pekic SQS (1995). Identification of QTL for drought responses in maize and their use in testing causal relationships between traits. J. Exp. Bot. 46: 853-865.

Le Deaux JR, Graham GI, Stuber CW (2006). Stability of QTLs involved in heterosis in maize when mapped under several stress conditions. Maydica, 51: 151-167.

Loo JA (1991). Statistics of Chi-square: $X^{2}$. J. Stat. 63: 2488-2499.

Paterson AL (1988). Resolution of quantitative traits into mendelian factors by using a complete linkage map of restriction fragment length polymorphisms. Nature 335: 721-726.

Quan R, Shang M, Zhang H, Zhao Y, Zhang J (2004). Engineering of enhanced glycine betaine synthesis improves drought tolerance in maize. Plant Biotechnol. J. 2: 477-486.

Ribaut J, Jiang C, Gonzalez-de-Leon (1997). Identification of quantitative trait loci under drought conditions in tropical maize. 2. Yield components and marker-assisted selection strategies. Theor. Appl. Genet. 94: 887-896.

Ribaut JMD, Gonz LC (1997). In Identification and transfer of ASI quantitative trait loci (QTL): A strategy to improve drought tolerance in maize lines and populations. (). Paper presented at the In Edmeades GO, M. 392-395. Mexico, D.F.: CIMMYT.

Ribaut JM, Hoisington DA, Deutsch JA, Jiang C, Gonzalez-de-Leon D (1996) Identification of quantitative trait loci under drought conditions in tropical maize. 1. Flowering parameters and the anthesis-silking interval. Theor. Appl. Genet. 92: 905-914.
Sari-Gorla M, Di FN, Villa M, Frova C, Krajewski P (1999). Genetic analysis of drought tolerance in maize by molecular markers. II. Plant height and flowering. Theor. Appl. Genet. 99: 289-295.

Sibov ST, de SC, Garcia AA, Silva AR, Garcia AF, Mangolin CA (2003). Molecular mapping in tropical maize (Zea mays L.) using microsatellite markers. 2. Quantitative trait loci (QTL) for grain yield, plant height, ear height and grain moisture. Hereditas, 139: 107-115.

Tuberosa R, Salvi S, Sanguineti MC, Landi P, Maccaferri M, Conti S (2002). Mapping QTLs regulating morpho-physiological traits and yield: case studies, shortcomings and perspectives in droughtstressed maize. Ann. Bot. 89: 941-963.

Veldboom LR (1996). Genetic mapping of quantitative trait loci in maize in stress and nonstress environments: I.Grain yield and yield components. Crop Sci. 36: 1310-1319.

Wang CT, Li SK, Han BS (2006). The Participatory Appraisal on the Main Constraints for the Realization of Corn Yield Potential. China Soft Sci. 7: 53-59.

Wang SC, Basten CJ, Zeng ZB (2005). Windows QTL cartographer 2.5 user manual. North Carolina State University, Bioinformatics Research http://statgen.ncsu.edu/ shchwang/WinQTLCart.pdf.

Xiao Y, Xin-Hai L, Shi-Huang Z, Xiang-Dong W, Mng-Shun L, YongLian Z (2004). Identification of Quantitative Trait Loci (QTLs) for Flowering Time Using SSRMarker in Maize under Water Stress. Korean J. Genet. 26: 405-413.

Yu J, Arbelbide M, Bernardo R (2005). Power of in silico QTL mapping from phenotypic, pedigree, and marker data in a hybrid breeding program. Theor. Appl. Genet. 110: 1061-1067.

Zeng Z (1994). Precision mapping of quantitative trait loci. genetics, 136: $1457-1468$.

Zhang ZM, Zhao MJ, Ding HP, Rong TZ, Pan GT (2006). QTL mapping analysis of plant height and ear height of maize (Zea mays L.). Genetika, 42: 391-396. 